\title{
Flow Effects due to Pulsation in an Internal Combustion Engine Exhaust Port
}

\author{
Bernhard Semlitsch ${ }^{\mathrm{a}, *}$, Yue Wang ${ }^{\mathrm{b}}$, Mihai Mihăescu ${ }^{\mathrm{a}}$ \\ ${ }^{a}$ Linné FLOW Center - Competence Center for Gas Exchange (CCGEx), Department of Mechanics, Royal Institute of Technology (KTH), Osquars \\ Backe 18, Stockholm, 10044, Sweden \\ ${ }^{b}$ National Key Laboratory of Aerodynamic Design and Research, Northwestern Polytechnical University, Youyi West Road, No. 127, Xi'an, \\ Shaanxi, 710072 ,China
}

\begin{abstract}
In an internal combustion engine, the residual energy remaining after combustion in the exhaust gasses can be partially recovered by a downstream arranged device. The exhaust port represents the passage guiding the exhaust gasses from the combustion chamber to the energy recovering device, e.g. a turbocharger. Thus, energy losses in the course of transmission shall be reduced as much as possible. However, in one-dimensional engine models used for engine design, the exhaust port is reduced to its discharge coefficient, which is commonly measured under constant inflow conditions neglecting engine-like flow pulsation. In this present study, the influence of different boundary conditions on the energy losses and flow development during the exhaust stroke are analyzed numerically regarding two cases, i.e. using simple constant and pulsating boundary conditions. The compressible flow in an exhaust port geometry of a truck engine is investigated using three-dimensional Large Eddy Simulations (LES). The results contrast the importance of applying engine-like boundary conditions in order to estimate accurately the flow induced losses and the discharge coefficient of the exhaust port. The instantaneous flow field alters significantly when pulsating boundary conditions are applied. Thus, the induced losses by the unsteady flow motion and the secondary flow motion are increased with inflow pulsations. The discharge coefficient decreased about $2 \%$ with flow pulsation. A modal flow decomposition method, i.e. Proper Orthogonal Decomposition (POD), is used to analyze the coherent structures induced with the particular inflow and outflow conditions. The differences in the flow field for different boundary conditions suggest to incorporate a modeling parameter accounting for the quality of the flow at the turbocharger turbine inlet in one-dimensional simulations.
\end{abstract}

Keywords: Internal combustion engines, Fuel economy, Turbocharged engines, Exhaust gas energy, Automotive exhaust systems

\section{Introduction}

Approximately $20-40 \%$ of the total provided energy after combustion is lost in the exhaust gasses [1,2]. However, the residual energy content of the exhaust gasses can be split into two parts, thermal energy and pressure energy. The exhaust gasses in the cylinder after combustion are hot and the gasses contain therefore potential usable energy, which can be extracted in a further process [3]. Thus, turbocharger are often used to expand in part the heat and the pressure potential [4], in order to increase the specific engine efficiency.

The exhaust stroke of a four stroke engine can be divided into two main periods, the blow-down and the

\footnotetext{
${ }^{*}$ Tel. +4687907157 / Fax: +4687969850

Email address: bernhard@mech.kth.se (Bernhard Semlitsch)
}

scavenging pulse. The pressure difference between the in-cylinder pressure and the pressure in the exhaust tract forces the exhaust gas expulsion at the early stage, which is referred as the blow-down phase. When the valves start to open, only a small slid between the valve head and the valve seat represents the discharge passage. Nevertheless, during the blow-down phase, the mass flow rate is high and decreases rapidly with the pressure dropping in the cylinder pod. Thereafter, when the pressure difference between the cylinder chamber and the exhaust manifold is balanced, the scavenging phase takes place, in which the piston cleans out the rest of the residual gas by its motion towards the top dead center. During this phase, the mass flow rate is lower than during the blow-down phase. However, the response of the mass flow rate during the scavenging phase depends highly on the pressure conditions down- 
stream in the collecting manifold, the possible interactions with other cylinders over the manifold, and the back-pressure provoked by the turbocharger.

The turbine of the turbocharger extracts flow energy and generates thereby a resistance to the flow downstream of the exhaust port. The flow resistance induces a back-pressure, which complicates the gas expulsion from the cylinder since this results in additional work demanded from the piston. In order to reduce the back-pressure effect of the turbocharger, several approaches can be employed. A waste gate to bypass the turbocharger during the scavenging phase can be used to reduce the back-pressure. With variable valve actuation and divided exhaust periods two separated exhaust ports are used for the blow-down phase and the scavenging phase, where the valves are actuated at different times [5]. Variable valve timing, i.e. regulating adaptively the valve opening time, the opening duration, and the valve lift, is crucial for the engine performance [6] and can be used to reduce the fuel consumption of the internal combustion engine [7]. However, the alteration of the valve timing influences the emission production. Simulating the entire process of an internal combustion engine with all degrees of freedom is computationally unaffordable. Therefore, fast simplified models are utilized for the initial design process of the internal combustion engine [8]. However, the physics and the behavior of the engine must be represented by the model. An oversimplified model may not represent the real engine any more. The inflow conditions into the turbocharger turbine can crucially effect its performance [9]. Usually, the valve timing and the valve opening-speed is studied and optimized by one-dimensional simulations, where the quality of the flow field and therefore the inflow conditions into the turbocharger turbine are not accounted for.

In one-dimensional modeling of the internal combustion engine process, tables, databases, and operation maps are used to describe the performance behavior of the individual constituents, such as the piping system, constrictions, or bends $[10,11]$. The tables or databases are based on empirical formulas with experimentally evaluated coefficients. For the exhaust port, the discharge coefficient is evaluated and employed in the onedimensional engine analysis to evaluate the total pressure drop [12]. A so-called flow bench experiment is performed, where the exhaust port discharge coefficient is evaluated at fixed valve lifts and constant specified total pressure drops. These experiments are usually performed on the real cylinder head at room temperatures. Hence, the flow streaming through the port is cold in contrast to a real engine case [13]. The discharge coef- ficient of sharp edged flow constrictions was evaluated experimentally [14], with the conclusion that the steady discharge coefficients are generally lower than those under unsteady flow conditions. The effect of pulsating flow on the exhaust port flow coefficients has been investigated numerically for different geometries regarding the blow down pulse and room temperatures [15]. It was found that the maximum difference in the flow coefficient was at most a $6 \%$ increase or a $7 \%$ decrease. The averaged flow coefficient over several valve lifts varied between $0.5 \%$ to $2.5 \%$ for the different geometries, which resembles a low impact on the total engine performance. Therefore, Bohac and Landfahrer [15] conclude that flow bench measurements at constant valve lift with constant mass flow rate are adequate.

Continuous ordinary differential equations can be used in one-dimensional simulation codes for more accurate modeling of engine components [16] or power plants [17]. The transfer functions for a given geometry can be obtained by impulse excitation to determine the characteristics of an engine transmission component. An experimental approach relating the mass flow rate with the dynamic pressure fluctuations can be used to abstract an ordinary differential equation model for an engine part [18]. A flow based model can be obtained reducing the Navier-Stokes equations via Galerkin projection onto representative flow modes [19]. Thus, the original governing system of partial differential equations is replaced by a set of ordinary differential equations, which are computationally inexpensive to compute. The Proper Orthogonal Decomposition (POD) method decomposes the flow field into a series of modes, where the modes are constructed such that the least number of modes is needed to reproduce the kinetic energy of the flow field [20]. The POD modes are also used to characterize the flow [21] and identify coherent structures with large energy contend. Also Fourier mode decomposition or dynamic mode decomposition have been used for constructing reduced order models [22], where certain frequencies are important [23].

The experimental assessment of the flow field in a complex, confined geometry, such as the exhaust port, is a challenging task [24]. However, experimental flow visualizations of the flow field development inside complex geometries, such as a closed cylinder with moving piston and combustion, have been performed [25]. Further, experimental flow visualization in an exhaust port can be performed with a considerable effort and assumptions [26], such as fixed valve lifts. Nevertheless, numerical computations of the flow in a complex geometry with non-moving boundaries can be easily per- 
formed. The computational effort of a numerical simulation depends on the complexity, amount of modeling and flow scales considered. A time-averaged simulation approach, such as steady state Reynolds Averaged Navier-Stokes (RANS) simulations, can be achieved with a rather low computational afford. However, the required time resolution of the flow phenomena for POD is not computed. To resolve all flow scales, i.e. Direct Numerical Simulation (DNS), is computationally expensive and the present purpose does not justify this the excessive usage of resources. With a reasonably resolved LES simulation, a substantial proportion of the inertial subrange in the turbulence spectra is resolved and only the smallest flow scales are modeled. The smallest flow scales dissipate the flow energy to heat and this behavior is of universal character, independent of the geometry. Generally, large energetic turbulent structures imply a substantial proportion of turbulent dissipation [27]. Hence, the LES approach reassembles a suitable trade between accuracy, reliability, and effort, which has been successfully applied to optimize the port geometry of internal combustion engines [28]. Many numerical investigations analyze the flow in the intake port and the consequences on the in-cylinder flow. Additionally, the flow in the ports of an internal combustion engine with pulsating boundary conditions has been simulated, e.g. [28]. However, the most studies emphasize on the computation models, performance parameter, or optimization of the geometry rather than giving insight into the generated flow structures occurring in the exhaust port.

This analysis is part of a larger investigation study treating the gas exchange process in an internal combustion engine. Within this paper the importance of dynamic boundary conditions on the flow generated losses in a realistic exhaust port of an internal combustion engine is investigated numerically. The three-dimensional compressible Navier-Stokes equations are simulated using an LES approach. A comparison of two cases, i.e. using constant inflow and outflow boundary conditions, and using engine cycle dependent inflow boundary conditions, at a constant valve lift are performed. These assumptions allow to isolate the effects caused due to flow pulsation. Therefore, the focus of contrasting the cases is held on the quantities, which are characteristic for the flow induced losses, as e.g. turbulent dissipation and friction losses. The change of the coherent flow structures are analyzed using the POD method.
Table 1: Specifications of the internal combustion engine, the SCANIA D12.

\begin{tabular}{lrl}
\hline \hline parameter & value & unit \\
\hline Displacement & 11.7 & $\mathrm{dm}^{3}$ \\
Bore & 127 & $\mathrm{~mm}$ \\
Stroke & 154 & $\mathrm{~mm}$ \\
Conn. rod length & 255 & $\mathrm{~mm}$ \\
Compression ratio & 18 & - \\
Exhaust valve opening & 136 & $\mathrm{CAD}$ \\
Exhaust valve closing & 359 & $\mathrm{CAD}$ \\
Valve head diameter & 41 & $\mathrm{~mm}$ \\
\hline \hline
\end{tabular}

\section{Case description}

The exhaust port geometry studied in this numerical investigation stems from a real internal combustion engine, the SCANIA D12, including the cylinder and the exhaust port valves. The relevant specifications of the internal combustion engine are tabulated in Tab. 1. The geometry used for the computational simulations is depicted in Fig. 1. On top of the engine cylinder, two exhaust ports are situated off-center, each in a quarter section of the circular cylinder, forming the outlet from the cylinder. The two valve ports conduct two bends from the vertical attachment into horizontal direction and merge together into one pipe. Valves are situated in each of the ports, performing opening and closing motion during the engine cycle. For the simulations, the valves are fixed at a valve lift of $5 \mathrm{~mm}$, representing a low valve lift compared to the maximum valve lift. The valve shape is shown in a frontal cut view through the exhaust port in Fig. 1 (C). The pipe bends exhibit a complex shape, bending in several directions to facilitate the valve actuation, which can be seen in Fig. 1 (B). After the conjunction $(y=0)$, an exit pipe $\left(d_{e}=42 \mathrm{~mm}\right)$ is attached to smoothly advect the flow structures out of the investigation domain. The length of the exit pipe spans ten exhaust pipe diameters downstream until the final outlet of the computational domain.

Laboratory-like boundary conditions are used to simulate the gas outtake process, in order to mimic a flow bench experiment. Thus, the ambient temperature of $293 K$ is applied at the outlet and a total temperature of $293 K$ is used at the cylinder inlet. At all walls, adiabatic wall boundary conditions and no-slip boundary conditions for velocity are applied. A non-pulsating case is defined by using a constant mass flow of $0.1 \mathrm{~kg} / \mathrm{s}$ at the inlet of the cylinder and a constant static pressure of 100 $k P a$ at the outlet. A pulsating case is defined by apply- 


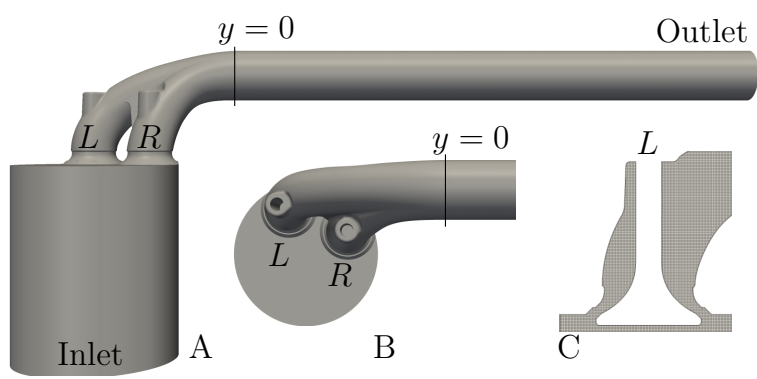

Figure 1: The analyzed geometry relevant for investigation of the gas outtake process is shown. A: the front view; B: the top view; $\mathrm{C}$ : the cross section of the $L$ valve.

ing a modulated mass flow rate at the numerical inlet plane of the cylinder, reassembling the piston motion, and a constant static pressure distribution of $100 \mathrm{kPa}$ at the outlet. As described before, the exhaust cycle of an internal combustion engine can be split into two phases, the blow-down pulse and the scavenging phase. Thus, the initial blow-down pulse was modeled by the function $\dot{m}_{c y l}=(0.04 \cos (100 \pi t)+0.12) \mathrm{kg} / \mathrm{s}$ in the time interval $0<t \leq 0.01$ for each pulse. The following scavenging phase was modeled by a constant mass flow of $0.08 \mathrm{~kg} / \mathrm{s}$ in the time interval $0.01<t \leq 0.02$ for each pulse. Thereafter, the next blow-down pulse follows the scavenging phase in a repetitive manner. Thus, the exhaust stroke period length $\tau$ is $0.02 s$ and the average mass flow is kept equal to the constant mass flow case. The applied variable mass flow rate at the computational domain inlet is plotted in Fig. 2.

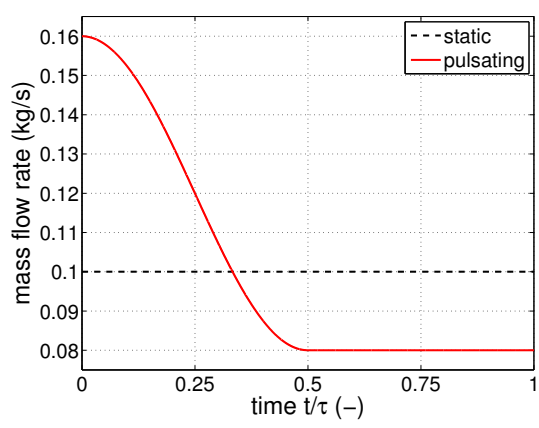

Figure 2: The variable mass flow rate $(\mathrm{kg} / \mathrm{s})$ applied as boundary condition at the inlet for the dynamic boundary condition case is depicted and contrasted with the constant mass flow rate of $0.1 \mathrm{~kg} / \mathrm{s}$.

\section{Simulation procedure}

\subsection{Numerical method}

The numerical computations are performed using the commercial finite volume code StarCCM+ by CD-
Adapco simulating the three-dimensional compressible Navier-Stokes equations. A wide range of turbulent flow scales occur in the flow of an exhaust port, since high velocity flow scales are involved in the turbulence generation process. Solving all turbulent flow scales occurring in the present problem would be computationally expensive. The most kinetic energy is contained by the spatially large flow structures. The kinetic energy is transferred from the large flow structures to the smaller flow structures via the turbulent energy cascade. At the smallest flow structures, the energy is dissipated into heat, which is of universal character at a distance from walls. In order to restrict the numerical effort to an adequate amount, only a substantial range of turbulent scales has been spatially resolved within the numerical mesh grid. This approach is commonly known as LES. Thus, the internal subrange of the turbulent spectra has been resolved within the mesh resolution used at least one order of magnitude capturing the $-5 / 3$ turbulence energy cascade. Hence, Favre-filtering has been applied to the solved set of governing equations. The flow scales smaller than the mesh-cell size are the so-called subgrid scales and represented by the residual stress tensor, which needs to be modeled. Modeling approaches can be applied using assumptions for the dissipative nature of these scales. However, for this complex flow situation no general valid assumptions can be made. Hence, no explicit subgrid scale model has been used in the current simulations. However, the numerical dissipation of the discretization scheme has been used to reassemble necessary subgrid scale dissipation. The simulated form of the Navier-Stokes equations can be written as,

$$
\frac{\partial\left(\rho u_{i}\right)}{\partial t}+\frac{\partial\left(\rho u_{i} u_{j}\right)}{\partial x_{j}}=-\frac{\partial p}{\partial x_{i}}+\frac{\partial \sigma_{i j}}{\partial x_{j}},
$$

where $t$ is the time, $x_{i}$ are the spatial coordinates, $\rho$ is the fluid density, $u$ is the velocity vector, $p$ is the static pressure, $\sigma_{i j}$ is the viscous stress tensor. The viscous stress tensor is defined as,

$$
\sigma_{i j}=\mu\left(2 S_{i j}-\frac{2}{3} S_{k k} \delta_{i j}\right),
$$

where

$$
S_{i j}=\frac{1}{2}\left(\frac{\partial u_{i}}{\partial x_{j}}+\frac{\partial u_{j}}{\partial x_{i}}\right)
$$

and $\delta_{i j}$ is the Kronecker-delta function. The conservation of mass and energy is guaranteed, by solving the conservation equations 


$$
\frac{\partial \rho}{\partial t}+\frac{\partial\left(\rho u_{i}\right)}{\partial x_{i}}=0,
$$

and

$$
\frac{\partial\left(\rho e_{0}\right)}{\partial t}+\frac{\partial\left(\rho e_{0} u_{j}\right)}{\partial x_{j}}=-\frac{\partial\left(p u_{j}\right)}{\partial x_{j}}-\frac{\partial q_{j}}{\partial x_{j}}+\frac{\partial\left(u_{i} \sigma_{i j}\right)}{\partial x_{j}},
$$

where $q_{j}$ represents the heat flux. The heat flux is calculated applying Fourier's law. The ideal gas law is applied to relate the static pressure with the static temperature and the fluid density, where the flowing media is assumed to be air. The isentropic exponent $\gamma$ was set to 1.4. The specific total energy is related to the static pressure, fluid density and flow velocity by,

$$
e_{0}=\frac{p}{\gamma-1}+\frac{1}{2} \rho u^{2} .
$$

The used numerical grid is not capable to resolve the boundary layer at the walls, therefore, wall functions have been used.

For time integration a second order implicit temporal scheme with a constant time-step is used. A hybrid second order bounded central differencing scheme, based on the normalized variable diagram approach [29], is used for spatial discretization. The scheme uses a blend of second and first order upwind scheme for numerical stabilization when the boundedness of the central differencing scheme is not guarantied.

\subsection{Numerical mesh-grid}

The computational grid is constructed using Star$\mathrm{CCM}+$ with a homogenous isotropic hexahedral core mesh in the exhaust port with a cell-core size of $0.6 \mathrm{~mm}$. Two prism layers form the interface of the cell-core to the walls of the domain. The prism layers exhibit a total thickness of $0.3 \mathrm{~mm}$ and a cell stretching factor of 1.2. Additional mesh stretching is applied towards the final section of the outlet domain to damp reflections at the outlet. In total, the computational grid consists of approximately eleven million cells. The numerical mesh grid is shown in Fig. 3. A grid sensitivity study for this kind of case setup and a comparison to experimental data has been presented previously [30]. The grid sensitivity study revealed that minor differences occur in the velocity and turbulence kinetic energy line plots. Moreover, the numerically predicted pressure drops agreed with the air flow bench experiments within an error range of $2 \%$ for the present set-up. Further, a significant proportion of the inertial subrange is captured by the numerical grids and the simulations resolve flow frequencies up to $10 \mathrm{kHz}$. The same solver has been successfully used for similar engineering applications, i.e. a turbocharger compressor, and the results were verified by comparing the numerical results with Picture Image Velocimetry (PIV) data and experimental measurements of the pressure ratio and the compressor efficiency [31].

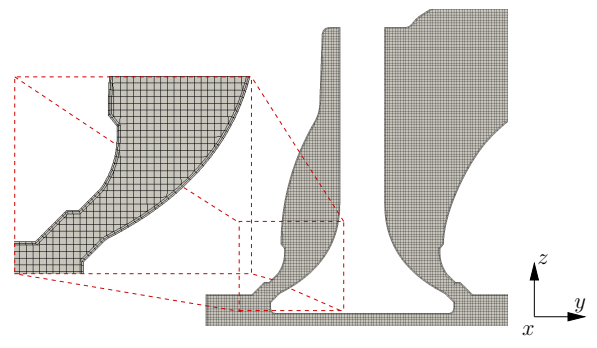

Figure 3: The computational mesh is illustrated in a cut-plane showing the $L$ valve.

\subsection{Modal flow analysis}

The largest flow structures are limited by the geometry extensions or the boundary conditions. Since the largest flow structures carry the highest kinetic energy, they dominate the characteristics of the flow field. A POD analysis is commonly used to identify large coherent structures in the flow. Hence, comparing the leading modes of a modal analysis from the fluid dynamic system indicates the effects of the boundary condition alteration.

A snapshot approach based on instantaneous samples of the velocity field has been used to perform the modal decomposition. For the modal analysis, only the section of interest is considered, since the numerical procedure requires a high amount of computational resources. Therefore, a small part of the cylinder and the two ports until the end of the conjunction is considered in the analysis. About 1300 snapshots of the instantaneous velocity field have been used, which were consecutively sampled at a time interval $\Delta t$ of $10^{-4} \mathrm{~s}$. Thus, flow phenomena in the range of $5 \mathrm{kHz}$ down to about $4.2 \mathrm{~Hz}$ are represented in the analysis, where the simulation resolves flow frequencies up to $10 \mathrm{kHz}$ with the used grid.

\subsubsection{Proper orthogonal decomposition analysis}

A short description of the Proper Orthogonal Decomposition (POD) method is introduced. For a more detailed description see Chatterjee et al. [20]. The POD method is the discretized, finite version of the 
Karhunen-Loéve theorem, where the Karhunen-Loéve theorem describes an optimal description of a stochastic process by an infinite series of orthogonal functions with random coefficients. However, with POD the flow field $u(x, t)$ is decomposed into temporal components $a_{j}(t)$ and spatial components $\Phi_{j}(x)$,

$$
u(x, t) \approx a_{0}(t) \Phi_{0}(x)+\sum_{j=1}^{J} a_{j}(t) \Phi_{j}(x) .
$$

The temporal components $a_{j}(t)$ are called time coefficients or the chrono modes and the spatial components are called the topo modes. The zeroth mode of the POD decomposition corresponds to the averaged mean flow field, while the higher modes represent the fluctuations to the mean flow field. The modes are selected such that an optimal representation, in least-squares sense, of the flow field is found,

$$
\min _{\Phi_{j}}\left(u(x, t)-\sum_{j=0}^{J} a_{j}(t) \Phi_{j}(x)\right)^{2},
$$

while the topo modes $\Phi_{j}(x)$ are required to be orthogonal.

The calculation of the discretized POD mode decomposition is based on a snapshot method. An ensemble $U=\left\{u_{1}, u_{2}, \ldots, u_{J}\right\}$ is formed by a number of snapshots of the velocity field sampled at a time $t_{k}$. The auto-covariance function averaged in time is calculated,

$$
r=\sum_{j=1}^{J} u_{j}^{T} w u_{j}
$$

where $w$ is a spatial weight for a numerical cell. The spatial weight is chosen to account for the change in cell volume when an inhomogeneous mesh is used. The optimization problem defined in Eq. 8 can be transformed into an eigenvalue problem using the auto-covariance function defined in Eq. 9,

$$
r \varphi_{k}=\lambda_{k} \varphi_{k},
$$

where $\varphi_{k}$ is the spatial eigenfunction and $\lambda_{k}$ is the associated eigenvalue. The eigenvalues are positive, real valued and represent the kinetic flow energy content of the mode [32]. The eigenvalue decomposition leads to an eigenvector, which represents the temporal description of the modes, the chrono modes. The spatial modes can be reconstructed with respect of the snapshot matrix $U$.

\section{Results}

In this section, the LES simulation results describing the flow field in the exhaust port are introduced. Firstly, the general features governing the flow with static boundary conditions are described. Following, the deviations from the original, non-pulsatile flow field with dynamic boundary conditions are discussed. Finally, the modal flow decomposition results and the discharge coefficients are presented.

\subsection{Constant mass flow rate case}

The cylinder is connected to two valve ports, which are equipped with a valve each. The purpose of the ports is to guide the flow out of the valve socket axis, in which the valves are mounted and actuated. The ports lead the flow into the exit pipe, where the two ports merge the flow. For this aim and due to spatial restrictions in an engine hood, the ports bend in a complex manner, which leads to a complex flow structure development in the exhaust port.

The valve ports are situated close to the outer periphery of the engine cylinder, as visible in Fig. 4 showing a top view of the cylinder. Moreover, a small proportion of the valve ports at the interface with the combustion cylinder is cut off due to the tight positioning. In Fig. 4, the time-averaged velocity magnitude contours are shown in a plane cutting through the valve ports and the valve stems. The flow field exhibits two lobes towards the cylinder walls due to the flow interaction with the cylinder walls during the exhaust process. In the later descriptions, the valve port closer to the outlet pipe is referred as the $R$ valve port, whereas the other valve port is referred as the $L$ valve port. Figure 4 (b) and Fig. 4 (c) illustrate the flow field development in the exhaust ports by showing in two cut-planes the timeaveraged velocity magnitude contours for the case of a constant mass flow rate applied on inlet, a constant static pressure at the outlet, and a fixed valve lift of 5 $\mathrm{mm}$. The two planes correspond to a vertical cut through the mid of the $L$ valve port, shown in Fig. 4 (a) (midlongitudinal plane) and a horizontal cut through the mid of the $R$ valve port, shown in Fig. 4 (b). Due to the higher pressure inside the cylinder compared to the ambient pressure, the flow is driven from the cylinder into the exhaust port. For a valve opening of $5 \mathrm{~mm}$, the geometry of the exhaust port and the valve seat represent a contraction with the shape of an annular Venturi channel. Thus, a non-axisymmetric conical jet forms when passing through the annular gap. In the further development, the annular jet contracts towards the valve stem 
and the annular jet stream is redirected towards the upper wall of the exhaust port. At the valve head, Görtlerlike vortical structures form due to the contracting jet streams and therefore, the jets do not remain attached to the valve head surfaces. A small wake forms in this region behind the valve stems close to the top wall and vortex shading takes place [30]. Further downstream, the flow follows the shape of the wall into the exit pipe.

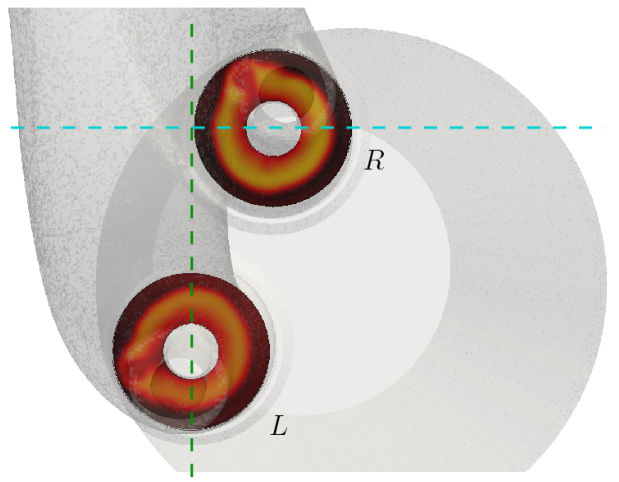

(a) top view

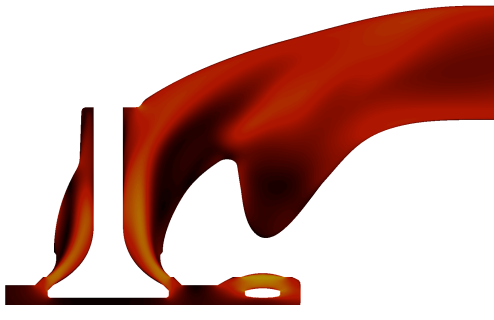

(b) $L$ valve port

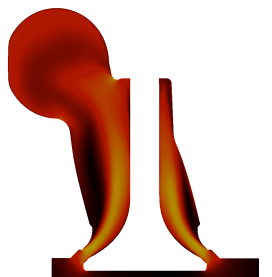

(c) $R$ valve port

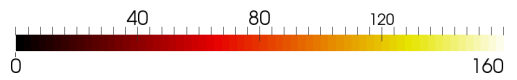

Figure 4: For the case of a constant mass flow rate representing the cylinder, the time-averaged velocity magnitude contours $(\mathrm{m} / \mathrm{s})$ in the individual valves are shown from a top view in (a), and in cut planes through the individual valve ports in (b) and (c). The location of the cut planes chosen for the $L$ and $R$ valve for further illustration are indicated by dashed lines.

The general flow field develops similarly in both ports. However, Fig. 4 exhibits that a higher velocity is observed in the $R$ valve port than in the $L$ valve port. The $R$ valve port is closer located to the outlet pipe than the $L$ valve port. Thus, the acting pressure gradient is higher in the $R$ valve port, which forces higher velocities in this port.

The valve port axes do not intersect with the centeraxis of the exit pipe. Therefore, two flow bends are necessary to lead the stream into the exit pipe. The curvature radius of the $L$ valve port is rather large, which causes that the flow bends smoothly into the exit pipe, as shown in Fig. 4 (b). The alteration of flow direction in the $R$ valve port is abrupt, compared to the $L$ valve port. The annular jet stream heads towards the top wall of the valve port, and there, the flow is deviated into the exit pipe, as shown in Fig. 4 (c).

With the formation of the annular jet in between the valve seat and the valve, the flow separates from the exhaust port walls. The flow separation causes the development of recirculation zones, which are indicated in Fig. 4 (b) and Fig. 4 (c). Additionally, flow separation at the valves can be observed, due to the formation of the Görtler-like vortical structures. However, at the outer shear layer forming in between the annular jet and the flow recirculation, high levels of turbulent kinetic energy occur due to the relative high velocity, as shown in Fig. 5. The induced secondary flow motion causes unwanted energy losses, due to dissipation of kinetic energy, which is shown in Fig. 5 (c-e). The turbulence dissipation rate in the $R$ valve port is higher than in the $L$ valve port.

The comparison of the Fig. 5 (a-b) and Fig. 5 (c-e) reveals that the turbulent kinetic energy distribution and the turbulent dissipation rate have a similar shape. Thus, where large coherent structures are induced, also a substantial amount of turbulence dissipation occurs.

The vortical structures generated in the exhaust port geometry are shown by iso-surfaces of the $\lambda_{2}$ criteria in Fig. 6 and three major zones of vortical structure production can be identified, i.e. the two shear layer of the annular jet and the mixing layer where the streams from the individual valve ports merge. With the flow separation at the valve port inlet, vortical structures are generated and shed in the manifesting shear layer. Further, a shear layer is formed in between the two streams (in the junction region, where the two streams from the two valve ports merge). Vortical structures are generated due to this shear layer instability, which are shown in Fig. 6. A few vortical structures can be seen at the top of the valve ports bending around the valve stems.

\subsection{Pulsating mass flow rate case}

With the pulsation of the inlet mass flow rate, the flow field exhibits an extended range of velocity regimes as compared to the constant mass flow rate case. The evolution of the velocity magnitude contours during a pulsation cycle is depicted in Fig. 7 for the $L$ valve port and in Fig. 8 for the $R$ valve port. Each cycle, the mass flow rate pulse is initiated at $t / \tau=0$ at the bottom of the cylinder. Thus, the response of flow field in the exhaust port is delayed and the maximum flow velocities in the valve port occur at about $t / \tau=0.5$. At the pulse peak, the velocity magnitudes in the exhaust port can reach up 


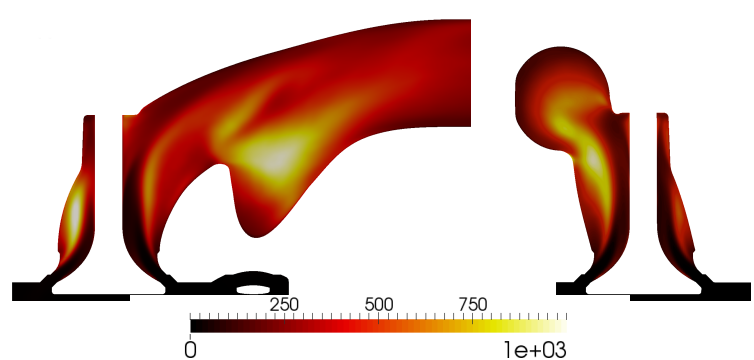

(a) $L$ valve port

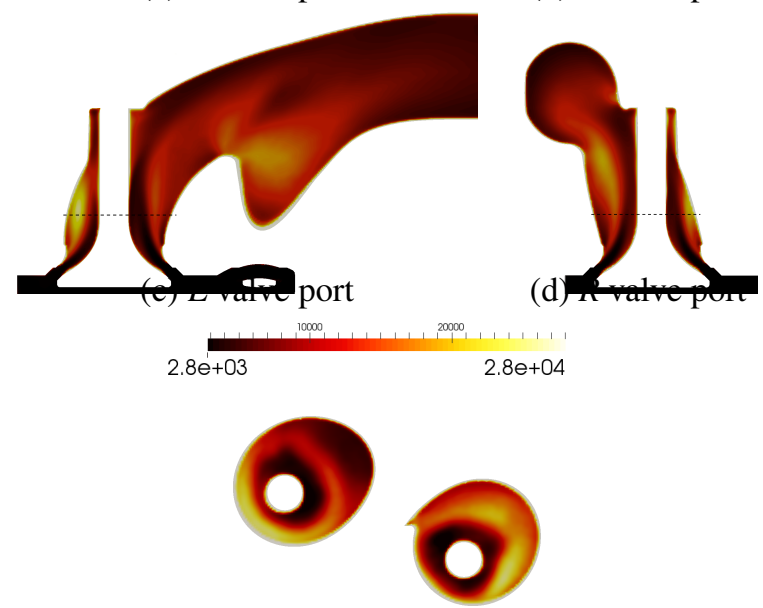

(e) horizontal cut-plane

Figure 5: The turbulence kinetic energy contours $\left(\mathrm{m}^{2} / \mathrm{s}^{2}\right)$ are shown in two cut-planes in Fig. (a) and (b). High turbulence kinetic energy can be seen in the region of the shear layer development of the annular jet rising at the valves. The turbulence dissipation-rate $\left(\mathrm{m}^{2} / \mathrm{s}^{3}\right)$ is illustrated in the Fig. (c), (d), and (e), where the plane location chosen for (e) is indicated as dashed lines in (c) and (d). A large amount of turbulence dissipation takes place on the port walls.

to $145 \mathrm{~m} / \mathrm{s}$, whereas for the constant mass flow rates, the velocity magnitudes in the exhaust port stay below 130 $\mathrm{m} / \mathrm{s}$. The components of total pressure losses have been investigated by Wang et al. [30]. Kinetic energy losses in the exhaust port scale quadratically with the velocity magnitude in the exhaust port. Thus, the increase of the velocity magnitude leads to enhanced total pressure losses during part of the cycle.

With the described flow separation from the valve port walls, recirculation regions arise between the annular jet and the valve port walls. When a constant mass flow rate is applied as inlet boundary condition, the separation bubble is rather static and does not exhibit large variations in size. However, with pulsating inlet boundary conditions, the separation zone changes the spatial extent over the pulsation cycle. Figure 8 illustrates the velocity magnitude contours in the $R$ valve port occurring at different stages of the pulsation cycle.

The distribution of the total pressure shown in Fig. 9

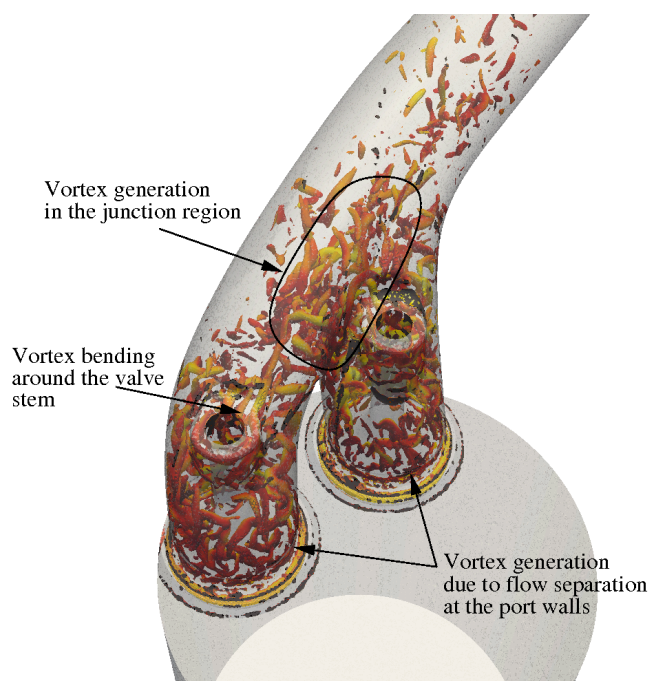

Figure 6: The iso-surfaces of the $\lambda_{2}$ criteria colored by the velocity magnitude are show for a time instant. Three major zones of vortical structure generation are emphasized, i.e. flow separation at the port walls and mixing layer formation in the junction region.

indicates the regions where flow energy is dissipated and lost for further exploitation. Comparing Fig. 4 with Fig. 7 (b), which are shown at the same scales, it can be observed that the peak velocities magnitudes are higher for the pulsating flow case. As mentioned, the most generation mechanisms of total pressure losses scale quadratic with the velocity. Thus, the total pressure losses are expected to be higher, when higher flow velocities occur. The instantaneous total pressure distributions for the two cases (i.e. static vs. pulsating) are compared in Fig. 9. Although, the total pressure is slightly higher in the cylinder for the pulsation cycle period $t / \tau=0.5$ (shown in Fig. 9 (c)), much lower total pressure values in the flow recirculation regions can be observed than with constant inflow boundary conditions. In the recirculation regions of the $L$ valve port, values below $96 \mathrm{kPa}$ can be observed for the pulsating mass flow rate case (see Fig. 9 (c)), while for the constant mass flow rate case the total pressure values stay above $100 \mathrm{kPa}$ (see Fig. 9 (e)). Nevertheless, analyzing Fig. 9 (c), one can see that the total pressure contours below $96 \mathrm{kPa}$ are coming from the region of the $R$ valve port. Further, the difference of the velocity magnitudes occurring in the two individual valve ports becomes obvious comparing Fig. 7 and Fig. 8. The largest differences between the velocity magnitudes is most apparent during sequences of flow acceleration $(t / \tau=0.25)$ and flow deceleration $(t / \tau=0.75)$.

At the end of the pulsation cycle $(t / \tau=0)$, a low static pressure distribution manifests in the valve port. 


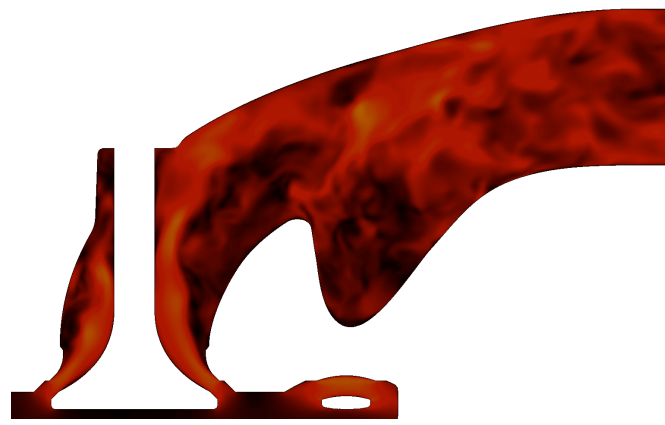

(a) $t / \tau=0$

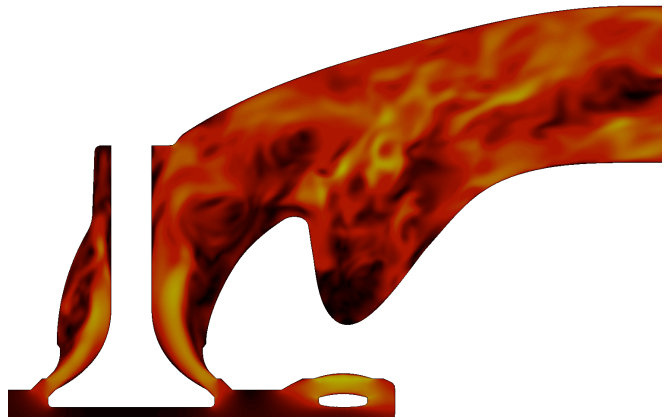

(b) $t / \tau=0.25$

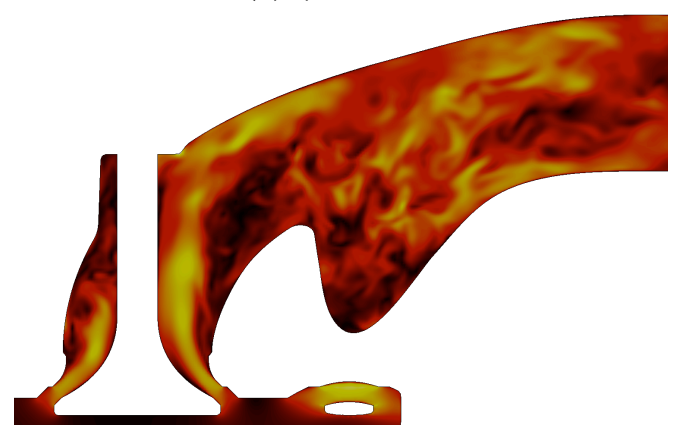

(c) $t / \tau=0.5$

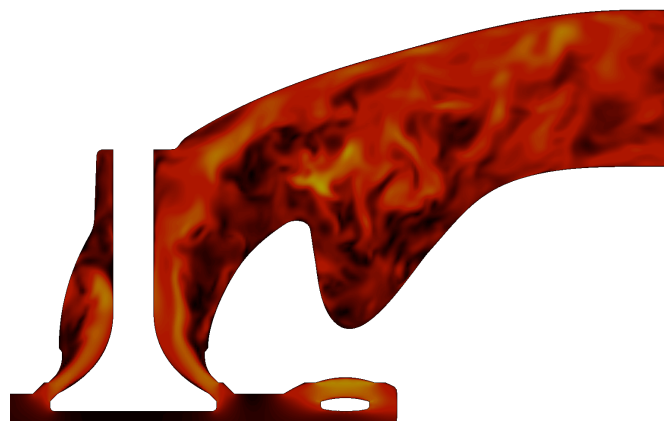

(d) $t / \tau=0.75$

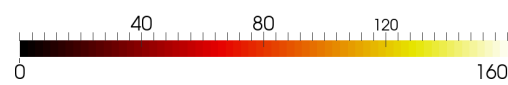

Figure 7: Four time-instants of the velocity magnitude $(\mathrm{m} / \mathrm{s})$ in a cutsection of the $L$ valve are shown when pulsating boundary conditions are applied. The pulse is initiates at $t / \tau=0$ at the bottom of the cylinder. (The scales have been kept equal as in Fig. 4.)

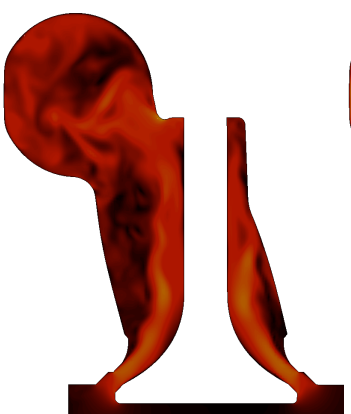

(a) $t / \tau=0$

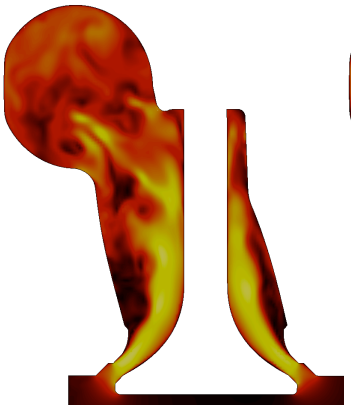

(c) $t / \tau=0.5$

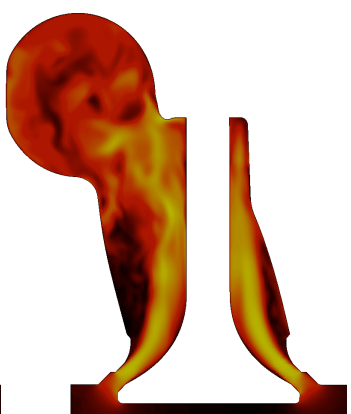

(b) $t / \tau=0.25$

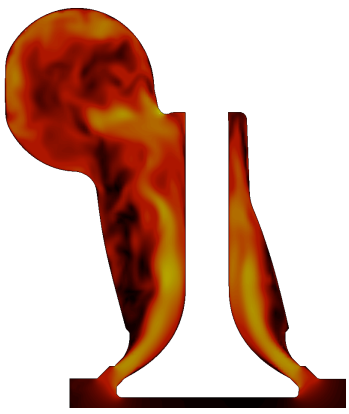

(d) $t / \tau=0.75$

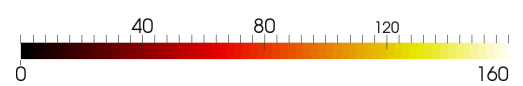

Figure 8: Time-instants of the velocity magnitude $(\mathrm{m} / \mathrm{s})$ in a cutsection of the valve port $R$.

With the peak of the mass flow pulse arriving in the narrow valve gap from the cylinder bottom $(t / \tau=0.25)$, the velocities in the annular jet are increased. The static pressure in the valve port does not adapt with the same rapid time scale, due to the enhanced entrainment of the annular jet at high velocities. The flow momentum generated with the pulse at the time period $t / \tau=0.25$ in the exit pipe remains draining fluid from the closer $R$ valve port at later times due to the conservation of flow momentum. The fluid is also provided by the region surrounding the annular jet. Consequentially, a low static pressure field is generated in the $R$ valve port at the time period $t / \tau=0.5$. Hence, sharp pressure gradients in the flow occur during parts of the pulsation cycle. An isosurface illustrating the shape of the low static pressure regions in the $R$ port is shown in Fig. 10. This phenomenon is an effect of the flow pulsation. The lower static pressure distribution drains the flow at higher velocities out of the cylinder. Thus, enhanced flow losses occur in this valve port with flow pulsation. Additionally, the low static pressure at the valve port causes a larger spread of the annular jet and increased amount of flow recirculation in the valve at later times $(t / \tau=0.5)$.

In Fig. 10, the main flow direction for the individual 


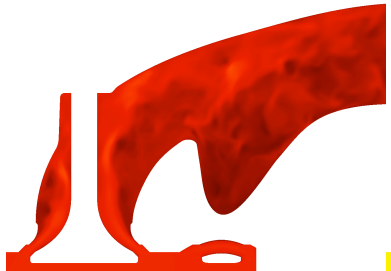

(a) pulsed $t / \tau=0$

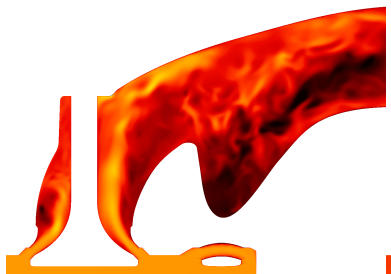

(c) pulsed $t / \tau=0.5$

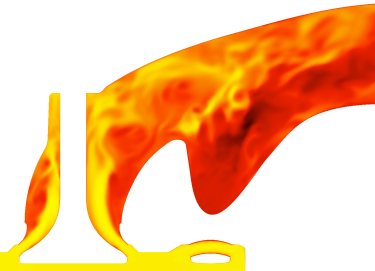

(b) pulsed $t / \tau=0.25$

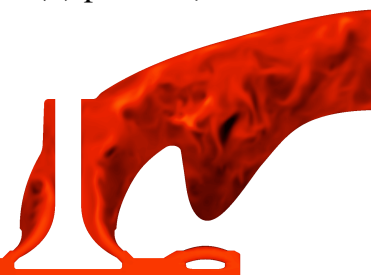

(d) pulsed $t / \tau=0.75$

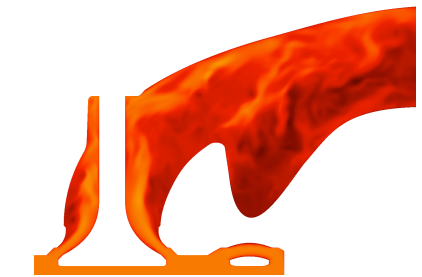

(e) constant
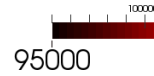

Figure 9: The total pressure distributions $(\mathrm{Pa})$ during a time instants for the two inlet boundary conditions are shown in the same scales. Higher gradients of the total pressure distribution, and thus higher losses, can be observed for the pulsating mass flow rate case at the time periods between $t / \tau=0.25$ and $t / \tau=0.5$.

port streams is indicated by color coded arrows. The stream from the $R$ valve port has a higher flow momentum than the stream originating from the $L$ valve port. In the junction region where the streams merge, the high momentum stream represents a barrier for the low momentum stream. Hence, the low momentum stream is forced to flow around the blocking stream. Figure 8 shows that the low momentum stream coming from valve port $L$ is driven towards the outer port walls. During different time periods, this effect is visible to varying extents. With that the low momentum stream from valve port $L$ flows towards the low pressure region generated. This induces a strong swirling motion in the exhaust pipe [30]. Due to the change of the low static pressure zone with flow pulsation, the swirling motion in the exhaust pipe is influenced.

\subsection{Results of the POD analysis}

The zeroth modes of both POD analysis correspond to the averaged flow field, where the shapes of the modes are shown in Fig. 11 (a) for the constant mass

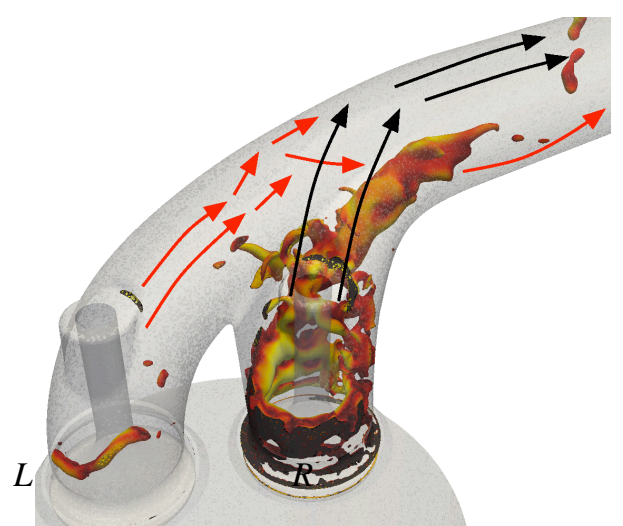

Figure 10: An iso-surface of the static pressure $(96 \mathrm{kPa})$ emphasizes the location of the low static pressure regions in the valve ports at the stage $t / \tau=0.5$ of the pulsation cycle. The arrows indicate the main flow direction of the individual valve port streams, i.e. colored in red and black for the $L$ and the $R$ valve port, respectively.

flow rate case and Fig. 11 (b) for the pulsed mass flow rate case. The shape of the two modes is similar and only minor differences, as e.g. a slight shift of the shear layer or a slight change of the distribution inside the separation bubbles, can be observed. Figure 12 shows the time coefficients of the two leading zero modes for the analyzed cases in a chosen time interval of four pulsation cycles. For the constant mass flow rate case, the time coefficient is approximately a constant. The oscillation of the time coefficient for the pulsating case corresponds to an amplification of the zeroth mode of about $25 \%$ at peak, compared to the time coefficient obtained for the constant mass flow rate. Further, the time delay of the pulse emitted at the bottom of the cylinder reaching the investigation region can be read off the plot shown in Fig. 12, since the time coefficient multiplied with the shape of the mode results in the flow field at a specific point in time. During a pulsation cycle, the flow field in the POD investigation domain reaches a minimum kinetic energy at about $t / \tau=0$ and a maximum kinetic energy close to $t / \tau=0.5$. The maximum increase in kinetic energy in the flow occurs at $t / \tau=0.25$ and the maximum decrease takes place around $t / \tau=0.75$.

The time coefficient of the zeroth POD mode for the static boundary condition case demonstrates a certain amount of fluctuations, which do not display an obvious pattern. However, for the pulsating boundary condition case, the zeroth chrono mode reveals a repetitive pattern of two small peaks in the beginning of each acceleration phase and two rather negligible hubs at the end of the acceleration phase. This peaks are only visible during the acceleration phase of the pulsatile flow, but not in the deceleration phase. Measuring the times when the peaks occur, a constant frequency of about $900 \mathrm{~Hz}$ between all 


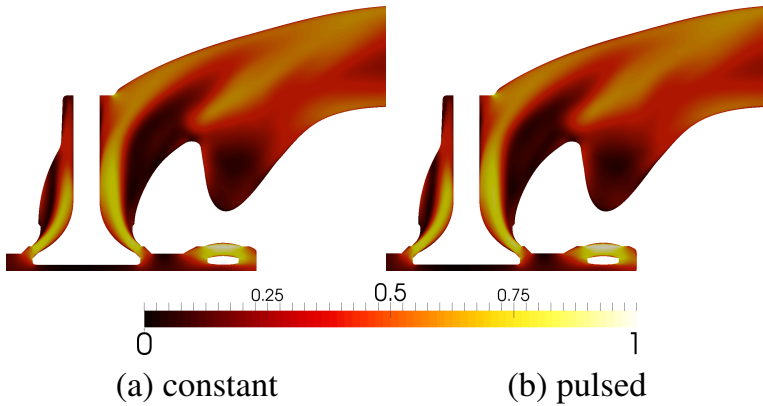

Figure 11: The normalized zeroth POD mode shape for both continuous and pulsed cases are presented, where the zeroth topo mode corresponds to the time-averaged flow field.

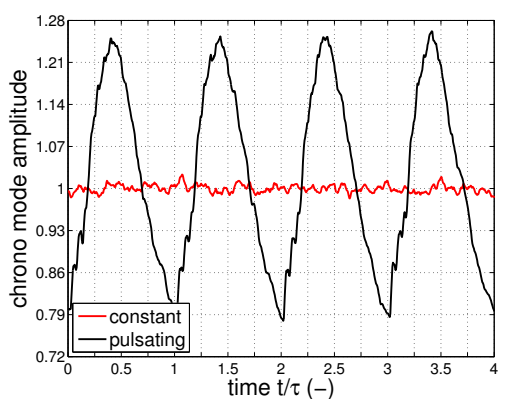

Figure 12: The time coefficients of the zeroth mode for both cases are shown, normalized by the mean amplitude for the constant boundary condition case.

peaks can be estimated.

The calculated POD eigenvalue of a specific mode is associated with the flow kinetic energy content as a fraction of the total flow kinetic energy. Figure 13 shows the kinetic energy distributions of the first twenty POD modes, representing the flow fluctuations to the zeroth POD mode, displayed for the cases using a static (a) and a dynamic (b) boundary condition. With a static mass flow rate boundary condition, the first POD mode contains approximately 1.9 percent of the fluctuating kinetic flow energy. The energy content represented by the POD modes decays initially rapidly, as the second POD mode contains approximately 1.4 percent and the third POD mode carries only 1 percent of the fluctuating flow energy. However, the energy contend decays firmly over the higher POD modes. The fifth POD mode contains about the half of the kinetic energy as the first POD mode. Using a pulsating mass flow rate at the inlet, the first POD mode carries slightly less kinetic energy (approximately 1.7 percent) than the first POD mode for the case with continuous mass flow rate boundary condition. However, the second and the third POD mode contain a higher proportion of the fluctuating flow en- ergy, compared to the POD modes obtained with constant mass flow rate boundary condition. For the higher POD modes the same firm decay in energy contend can be observed.

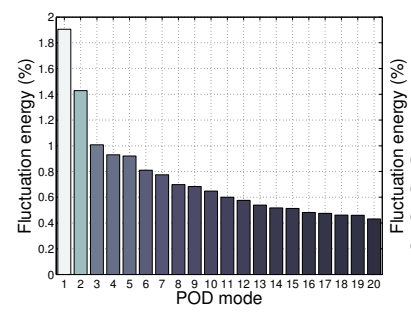

(a) constant

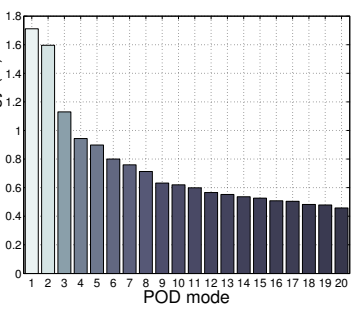

(b) pulsed
Figure 13: The fluctuation flow energy distribution of the first twenty POD modes is shown, for the static (a) and a dynamic (b) boundary condition.

Figure 14 compares the spectral properties of the first five POD chrono modes obtained with the two different inlet boundary conditions. The dominant frequencies in terms of kinetic energy are located in the low frequency range, independent of the boundary condition case. The pulsation frequency is approximately $50 \mathrm{~Hz}$ for the dynamic case and the dominant spectral peaks for the constant mass flow rate case occur at frequencies below this pulsation frequency. However, the low frequency peak for the constant boundary condition case is represented by the first three POD modes. The first POD mode for the constant boundary condition case is additionally associated to a frequency band of some hundred Hertz. The forth and the fifth chrono mode exhibit minor peaks in the range of 800 to $1000 \mathrm{~Hz}$.

With a pulsating inlet mass flow rate, the low frequency peak shifts to slightly higher frequencies and the peak amplitude is decreased, as shown in Fig. 14 (b). Further, this peak is also represented by the first three POD modes. Figure 14 (c) shows that the first two chrono modes exhibit a similar frequency content for the pulsating case. The following forth and fifth POD mode correspond to the peaks visible between 800 to $1000 \mathrm{~Hz}$, which are clearly enhanced compared to the constant mass flow case shown in Fig. 14 (a). These two modes exhibit similar spectral properties, as shown in Fig. 14 (d) for a chosen time interval. It can be observed that every peak of the fourth chrono mode is followed by a delayed peak of the fifth chrono mode. Thus, there occurs a phase shift between the two time coefficients. Nevertheless, the frequency of $900 \mathrm{~Hz}$ was already observed analyzing the zeroth chrono mode. 


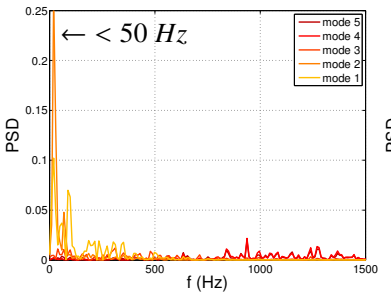

(a) constant

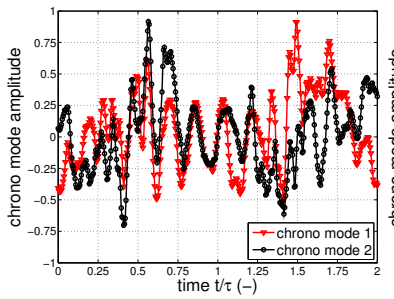

(c) chrono mode 1 and 2

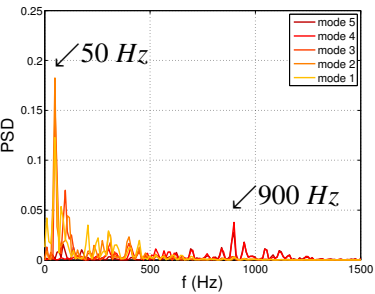

(b) pulsed

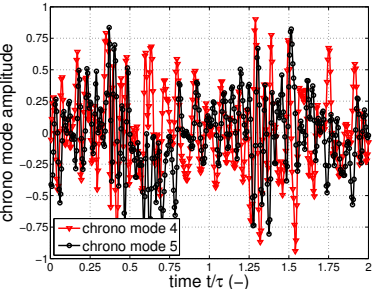

(d) chrono mode 4 and 5
Figure 14: The spectral properties of the first five chrono POD modes are shown, for the static (a) and a dynamic (b) boundary condition. The first and the second chrono mode (c), and the forth and the fifth chrono mode (d) of the pulsating case are plotted in a selected time interval. The spectral correlation of the two modes can be observed at low (c) and high (d) frequencies.

\subsubsection{Static mass flow rate inlet}

The shapes of the leading POD modes can often be associated with a physical mechanisms. Starting examining the topo modes obtained for a constant mass flow rate at the cylinder inlet, the first two POD modes can be associated with the unsteady flow motion in the individual valve ports, whereas the first mode is related to the unsteady flow motion in the $R$ valve port and the second mode is related to the unsteady flow motion in the $L$ valve port. The three following topo modes are linked with the mutual interaction of the two streams in the conjunction of the valve ports.

The first POD mode exhibits high magnitudes in the region of the $R$ valve port. Figure 15 illustrates the POD mode shape in two cross-sections. The topo mode shows that the mode is associated with the shear layer formation due to the flow separation of the annular jet from the $R$ valve port. Certain similarities can be observed comparing the shape of the turbulence dissipation rate shown in Fig. 5 (d) for the static mass flow rate case with the POD mode shape shown in Fig. 15 (a).

The complex three-dimensional distribution of the high magnitudes of the first POD mode is illustrated in Fig. 15 (c-d) by an iso-surface of the topo mode magnitude. At the top of the valve port $R$, Fig. 15 (c) shows that the wake behind the valve stem is part of this mode. It can be noted that the wake stretches over a small extent. One lobe of the first topo mode visualization can

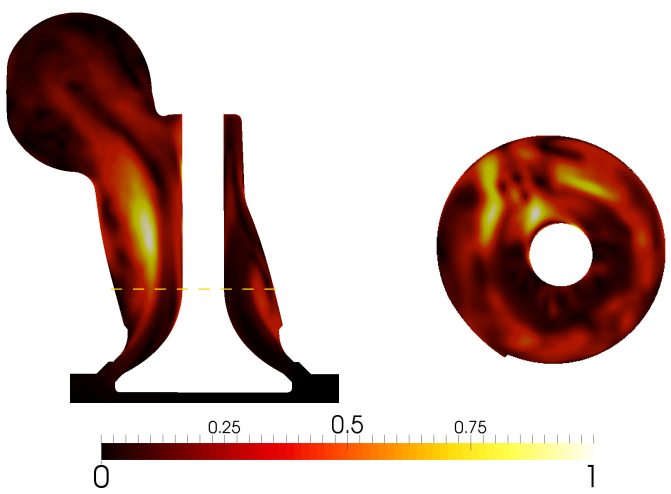

(a)

(b)

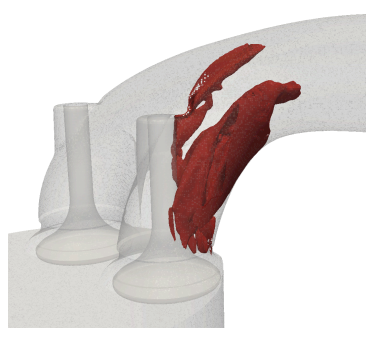

(c)

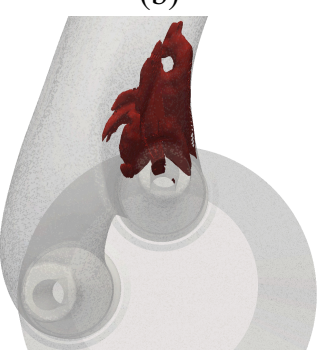

(d)
Figure 15: For the constant mass flow case, the first mode magnitude contours are illustrated. Two cross-sectional views across the $R$ valve are presented in (a) and (b), where (b) shows a horizontal cut at the dashed line indicated in (a). High magnitude values with the shear layer of the annular jet and the wake behind the valve stem can be seen. Figure (c) and (d) show an iso-surface of the mode magnitude. The mode can be associated with the shading behind the valve stem and the vortical structures generated at the walls within the separation zones.

be associated with the shear layer formed by the annular jet, which is also visible in Fig. 15 (b). The higher topo mode magnitudes in the annular jet shear layer occur towards the cylinder walls and towards the exit pipe. Another structure can be seen at the valve port wall close to the valve port conjunction, which originates from the velocity defect induced due to the close positioning of the valve port to the cylinder periphery. This structure can be seen clearly in Fig. 15 (b) showing a cross-sectional view. However, this POD mode cannot be strictly associated with one physical generation mechanism. Moreover, several mechanism occurring at different flow frequencies are related with this POD mode shape, as e.g. shedding behind the valve stem, and shear layer formation.

The second POD mode shape can be associated with the unsteady flow motion caused in the $L$ valve port. In Fig. 16 (a) and Fig. 16 (b) two cross-sectional views through the geometry of the $L$ valve port are shown, illustrating the regions of high magnitudes of the mode. 
Figure 16 (a) depicts high amplitudes of the topo mode with the formation of flow separation of the annular jet from the valve port. The side opposing the exit pipe exhibits higher mode magnitudes than other regions of the annular jet shear layer. This local increase is also visible in Fig. 16 (b), where the annular jet shear layer is emphasized in the POD mode magnitude contours. Comparing the POD mode shape shown in Fig. 16 (a) with the turbulent dissipation rate depicted in Fig. 5 (c), several distribution similarities can be noted. The turbulent dissipation rate exhibits higher magnitudes at the side opposing the exit pipe as the POD mode shape. However, the POD mode shape is interrupted in the shear layer of the annular jet opposing the exit pipe.

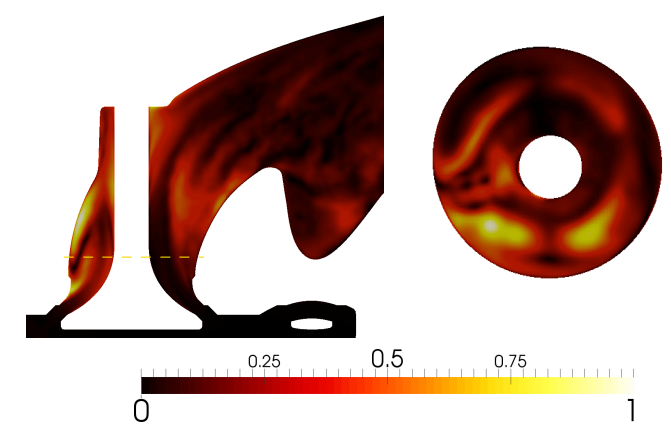

(a)

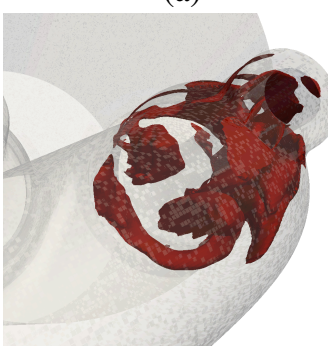

(c) (b)

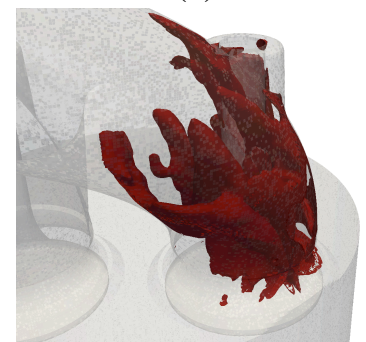

(d)
Figure 16: The second POD mode is shown. The mode is primary related to the $L$ valve port and the flow structures generated at the valve. In the cut-section through the $L$ valve port for the constant mass flow case, (a) shows the $L$ valve port plane and (b) a horizontal cut through the $L$ valve port, where the location is indicated by a dashed line in (a). (c) and (d) show an iso-surface of the mode magnitude for two different views.

A three-dimensional illustration of the second POD mode magnitude distribution is shown in Fig. 16 (c) and (d) by visualizing an iso-surface of the topo mode magnitude. At the top wall, the wake behind the valve stem is visible in the Fig. 16 (d). The extent of the wake formed behind the valve stem is small, since the wake is generated when the annular jet stops following the valve stem and bends towards the exit pipe. On the upper side of the valve, two lobes bend with the annular jet into the exhaust pipe, which originate from the outer edges of the valve. These large scale vortical structures have also been noted by Wang et al. [30] and the structures have been shown on a cut plane in terms of the streamwise vorticity. These structures can be considered as Deanlike vortices [33], which are induced due to the bending of the $L$ valve port.

The third POD mode shape exhibits high magnitudes in the lower region of the conjunction of the exhaust ports, as depicted in Fig. 17, which correspond to the mixing interaction of the two streams coming from those ports. Figure 17 (b) shows that the shape of the mode magnitude contours exhibits high magnitude values where the structures of the first POD mode reach into the conjunction. This third POD mode combined with the first POD mode results in a completing flow fluctuation representation of the large flow scales in the $R$ valve port, since the distribution is similar to the turbulence kinetic energy distribution shown in Fig. 5. The fourth and fifth POD mode describe a flow mixing behavior in the conjunction region of the exhaust ports, similar to the third POD mode.

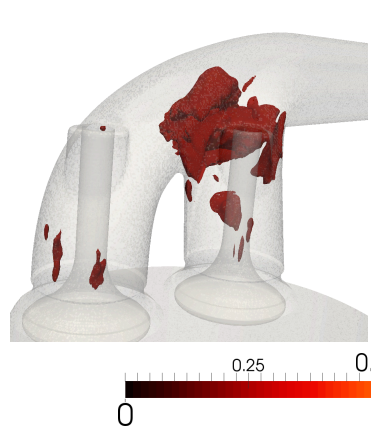

(a)

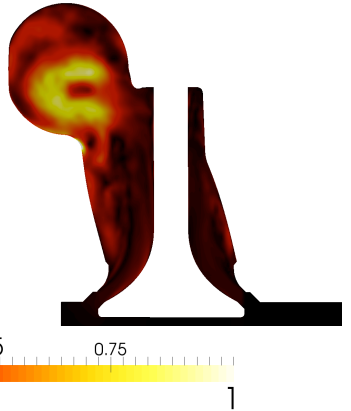

(b)
Figure 17: The iso-surface of the high magnitudes within the third POD mode are shown in (a). Figure (b) exposes the shape of mode by showing the contours in a cut-plane through the $R$ valve port.

\subsubsection{Pulsating mass flow rate inlet}

With the pulsating mass flow rate as inlet boundary condition, the first POD mode exhibits a similar shape as with the constant mass flow rate boundary condition. The wake behind the valve stem at the top wall of valve port $R$ is captured by this POD mode, as well as the structure generated due to the velocity defect provoked due to the close positioning of the valve port to the periphery of the cylinder. A part of the three-dimensional POD mode magnitude iso-surface, visible in Fig. 18 (a) and Fig. 18 (b), can be associated with the shear layer 
induced by the annular jet, where this POD mode shape stretches further towards the exit pipe than the first POD mode shape obtained with static boundary conditions.

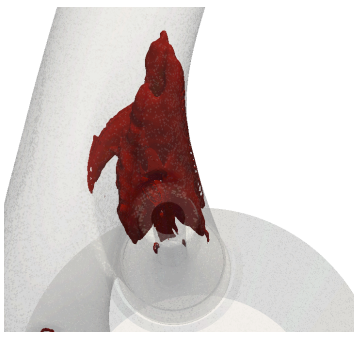

(a) $1^{s t}$ POD mode

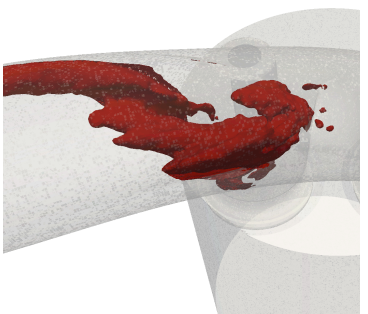

(c) $2^{\text {nd }}$ POD mode

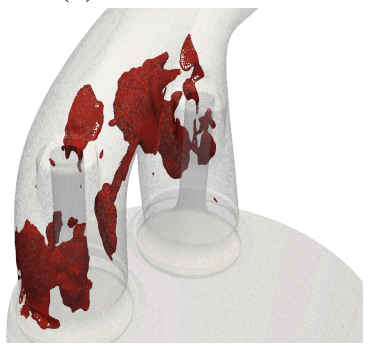

(e) $3^{\text {rd }}$ POD mode

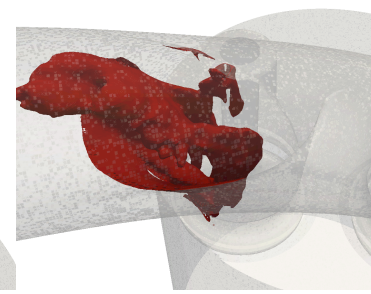

(b) $1^{\text {st }}$ POD mode

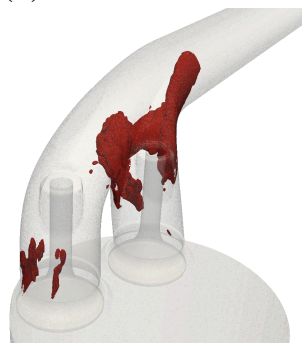

(d) $2^{\text {nd }}$ POD mode

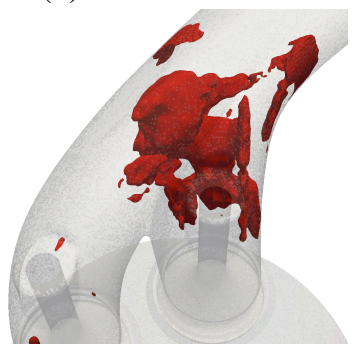

(f) $4^{\text {th }}$ POD mode
Figure 18: Iso-surfaces illustrate the shape of the leading POD modes obtained with pulsating boundary conditions.

The three-dimensional shape of the second POD mode is shown in Fig. 18 (c) and Fig. 18 (d). The locations of high topo mode magnitudes coincide with the region, where the mixing process of the two streams rising from the ports takes place. The shape of this POD mode exhibits high magnitudes in between where the first POD mode shape reveals high magnitudes. Nevertheless, the topo mode components of the two POD mode reveal opposite signs. By analyzing again Fig. 14 (b), one can see that the chrono modes of the first three POD modes exhibit a similar spectral content at a low frequency of $\sim 50 \mathrm{~Hz}$, which corresponds to the pulsation frequency imposed as boundary condition. The chrono modes of the first two POD modes were already compared in Fig. 14 (c). The interaction of the two modes represents a waving motion, which describes the phenomena of the pressure pumping in the $R$ valve port with pulsation boundary conditions as described before.

The POD analysis for the pulsating mass flow rate condition does not reveal a mode clearly corresponding to the unsteady flow motion in the $L$ valve port as it was shown for the constant mass flow case. However, the second and the third POD mode show high magnitudes in the mixing region nearby the junction of the ports and additionally, high magnitudes in the $L$ valve can be observed. As visible in Fig. 18 (e), the wake behind the valve stem in the $L$ valve port is captured in the third POD mode shape, as well as parts of the shear layer induced by the annular jet separation from the $L$ valve port. The fourth POD mode shape is shown in Fig. 18 (f) and this POD mode is responsible for the peak in the chrono mode spectra at approximately 900 $\mathrm{Hz}$. However, this POD mode iso-surface of high magnitudes manifests in the conjunction region, representing the mixing between the streams rising from the individual valve ports.

\subsection{Discharge coefficient}

The discharge coefficient $C_{D}$ is defined as the ratio of the real mass flow rate $\dot{m}_{\text {real }}$ to the ideal mass flow rate $\dot{m}_{\text {ideal }}$, which can be estimated by isentropic relations. Thus, the discharge coefficient quantifies the resistance generated within the geometry with respect to the theoretical ideal value,

$$
\begin{aligned}
C_{D}= & \dot{m}_{\text {real }} \frac{\sqrt{R T_{0}}}{A_{R} p_{0}}\left(\frac{p_{0}}{p_{T}}\right)^{\frac{1}{\gamma}} . \\
& \left(\frac{2 \gamma}{\gamma-1}\left[1-\left\{\frac{p_{T}}{p_{0}}\right\}^{\frac{\gamma-1}{\gamma}}\right]\right)^{-\frac{1}{2}},
\end{aligned}
$$

where $A_{R}$ is reference area, $R$ is the specific gas constant, $T_{0}$ is total temperature in the cylinder, $p_{0}$ is total pressure in the cylinder, and $p_{T}$ is the area averaged static pressure at the flow restriction. The reference area $A_{R}$ commonly used, is the so-called valve curtain area $A_{R}=\pi D_{v} L_{v}$, where $L_{v}$ is the valve lift and $D_{v}$ is the valve head diameter.

For static mass flow rate inflow boundary conditions, a discharge coefficient of 0.64 has been obtained, whereas for the pulsating mass flow rate inflow boundary conditions, a cycle averaged discharge coefficient of 0.62 has been calculated. A decrease of $1 \%$ in the discharge coefficient with the peak pulse arriving in the valve ports is observed. This difference between the static and pulsatile discharge coefficients obtained via the present simulations is in the range quoted by Bohac and Landfahrer [15]. The mass flow rate passing 
through the individual valve ports with flow pulsation is $46.9 \%$ and $53.1 \%$ for the $L$ and the $R$ valve port, respectively. However, the discharge coefficients through the individual valve ports differs less than $0.5 \%$ in cycle average.

\section{Conclusions and discussion}

The effect of pulsating flow conditions in an exhaust port of an internal combustion engine has been studied numerically, by simulating the governing compressible flow equations. Two LES simulation data sets have been compared, one using a constant mass flow rate as inlet boundary condition and a second using a pulsed mass flow rate as inlet boundary condition. Engine-like shaped pulses, representing the blowdown and the scavenging phases of the exhaust stroke of a four stroke engine, have been used to model realistic flow responses. A detailed flow description for the flow cases has been reported and a POD analysis has been performed, describing the most energetic flow structures. The discharge coefficient for the pulsatile flow was about $2 \%$ lower than for static mass flow rate boundary conditions applied at the inlet.

For this investigation, laboratory-like boundary conditions at room temperatures have been used, in order to approximate the conditions applied in an air flow bench experiment, which are typically performed in industry. After combustion, the exhaust gasses in the cylinder of the engine exhibit temperatures at approximately 1200 $K$ and pressures above $500 \mathrm{kPa}$ occur. Thus, the flow velocities are higher in a realistic engine and compressible flow phenomena are expected to take place. Additionally, the motion of the valve during the exhaust cycle has not been considered, which might have an influence on the flow field development in the exhaust port. In a four stroke engine, the exhaust cycle constitutes in onefourth of the total operation cycle in a combustion engine. Thus, only during approximately one-fourth of the operation cycle the exhaust gasses are pumped through the port, while in the rest of the cycle the flow is approximately standing.

Nevertheless, assumptions (cold, continuous flow at low pressure drops) have been made to mimic a flow bench experiment, which is commonly used to determine the discharge coefficient of the exhaust port employed in one-dimensional engine simulations. Therefore, the gradients of the primary variables are lowered compared to a realistic engine case. Using these assumptions, the effect of engine-like pulsatile flow can be analyzed isolated and studied systematically. The effect of flow pulsation had a significant impact on the pressure distribution compared to a static flow conditions throughout the exhaust port. Hence, this effect is expected to be enhanced during realistic engine conditions with steeper gradients. Thus, the basic observations of the flow dynamics are thought to be still valid in a real engine.

The inlet boundary condition was applied on the bottom of the cylinder. Thus, the pulse needs to propagate through the cylinder, before reaching the investigation region, i.e. the valve ports. With engine-like shaped pulsating mass flow rate boundary conditions, the velocities increase significantly during parts of the pulsation cycle compared to the flow velocities that occur with static mass flow boundary conditions. On the one hand, the increased flow velocities are enforced by the pulse as it was defined through the boundary conditions. On the other hand, the increased flow velocities result due to an observed flow pumping effect, which is caused by the flow acceleration and flow deceleration with the flow pulsation. At the end of a pulsation cycle, the flow decelerates in the port. Due to conservation of momentum the flow continues streaming and leaves due to conservation of mass a low the static pressure in the valve port. When the next mass flow rate pulse arrives with high momentum in the valve port, a high pressure difference accelerates the annular jet to higher velocities. These phenomena are captured by the first two modes of the POD analyses for the pulsating mass flow rate case.

Using POD analysis, the coherent flow structures with high kinetic energy content can be extracted. The difference of the characteristic flow structures occurring with different boundary conditions was highlighted by comparing the leading POD mode shapes obtained for both investigated cases. The time coefficients of the first three POD modes obtained with static boundary conditions exhibit a lower frequency than the engine-like pulsation frequency. Hence, flow features develop for the case with constant mass-flow rate at the inlet, which do not have the time to establish within engine-like pulsatile flow conditions. Thus, the observed flow features have generally different flow characteristics. The shape of the zeroth mode, corresponding to the mean flow field, exhibits a very similar pattern for both cases, the constant and the pulsating mass flow rate boundary condition. However, the time coefficients associated with the zeroth modes indicate that the mean flow field for pulsating case oscillates about $25 \%$ around the static boundary condition case. Further, the pulsating boundary condition case exhibited small peaks at the frequency of $900 \mathrm{~Hz}$ consistently during all the flow acceleration cycles. This indicates that pulsations impact the flow field substantially. 
Comparing the shape of the leading POD modes for the continuous and the pulsed cases suggests that the mixing losses are somewhat shifted from the annular jet separation region towards the conjunction region where the two streams rising through the ports mix. However, the valve ports are designed with a different length, which leads to altered velocities in the valve ports due to the different pressure gradients in the valve ports, and therefore, the pulses reach the conjunction with a phase shift. Hence, the velocity gradient between the streams is higher than compared with an exhaust port designed with equal valve ports lengths. The higher velocity gradient leads to an enhanced shear stress, which provokes higher kinetic energy losses. For the POD analysis of the pulsating boundary condition case, the forth POD mode shape manifests in the conjunction region of the valve ports. The spectral properties of this mode reveal peaks at $900 \mathrm{~Hz}$, which corresponds to small peaks in the time coefficient of the zeroth POD mode during the acceleration phase of the pulse. Approximately, the same time scale can be obtained by estimating the time delay between the two streams reaching the conjunction through the individual valve ports. The collected evidence pronounces that this POD mode describes the mixing loss, which has not been observed for the static boundary condition case. Thus, with flow pulsation, the mixing losses in the conjunction region are enhanced compared to the static boundary condition case.

In the $L$ valve port, two streamwise vortical structures rising from the valve head and bending into the exit pipe have been identified for the static boundary condition case. The amount of turbulent dissipation in the influence region of the streamwise vortical structures is lower compared to the values occurring in the other regions of the annular jet shear layer. Thus, these structures increase locally the fluid mixing process and decrease thereby the amount of back flow in the nearby separation bubble. Therefore, the velocity gradient between the annular jet and the separation is decreased, which results in reduced shear stresses in the shear layer and hence, less turbulent dissipation. However, the corresponding chrono mode reveals the low frequency properties of this structures, which are in a lower frequency range than the fundamental pulsation frequency for the pulsation case. Thus, with flow pulsation these structures might not develop, since the time scale is too short. Nevertheless, a chevron design at the outer periphery of the valve seat could force the development of such flow structures also under pulsatile flow conditions.

An influence of the tight positioning of the valve port with the periphery of the cylinder has been noted. A low velocity region establishes in the narrow gap between the valve and the port, which disturbs the formation of the annular jet. With the POD analysis, the induced flow structure could be shown for both valve ports and these structures are notable over a large distance. The flow velocity defect in the annular jet leads to streamwise orientated structures, which can be beneficial in some cases as described in the previous paragraph.

The observations based on the discharge coefficient estimations in the current investigation are similar to the observations made by Bohac and Landfahrer [15]. Bohac and Landfahrer [15] concluded in their study that static flow bench measurements are valid, since the averaged discharge coefficient over all valve lifts increases between $0.5 \%$ and $2.5 \%$. However, the current investigation shows that the flow field changes drastically with pulsating boundary conditions compared to static boundary conditions. The performance of a downstream located turbocharger depends on the quality of the inflow conditions to the turbocharger [34]. Hence, a parameter expressing the flow conditions upstream of the turbocharger inlet would be suitable to asses the performance of the energy recuperation device. Performing flow measurements under pulsatile inflow conditions to gather such a parameter might be challenging. Nevertheless, performing simple flow bench measurements applying a constant mass flow rate to approximate the discharge coefficient of an exhaust port, seams to be a crude approximation, since the occurring flow characteristics change with flow pulsation. Thus, for design optimization in terms of the exhaust tract and the valve timing, accounting for the engine like pulsations would be recommended to develop more energy efficient combustion engines. Additionally, low order modeling based on POD mode analysis considering more realistic flow boundary conditions would lead to better optimized engines.

\section{Acknowledgments}

This work was supported by the Competence Center for Gas Exchange (CCGEx) and the Swedish Energy Agency (STEM). The computational resources provided through the Swedish National Infrastructure for Computing (SNIC 2013/1-72) are greatly acknowledged.

\section{References}

[1] J. Fu, J. Liu, Y. Yang, C. Ren, G. Zhu, A new approach for exhaust energy recovery of internal combustion engine: Steam turbocharging, Applied Thermal Engineering 52 (1) (2013) 150 -159 . 
[2] M. He, X. Zhang, K. Zeng, K. Gao, A combined thermodynamic cycle used for waste heat recovery of internal combustion engine, Energy 36 (12) (2011) 6821 - 6829.

[3] T. Wang, Y. Zhang, J. Zhang, Z. Peng, G. Shu, Comparisons of system benefits and thermo-economics for exhaust energy recovery applied on a heavy-duty diesel engine and a light-duty vehicle gasoline engine, Energy Conversion and Management 84 (0) (2014) 97 - 107.

[4] N. Watson, M. Janota, Turbocharging the Internal Combustion Engine, London: The MacMillan Press Ltd., 1982.

[5] S. Gundmalm, A. Cronhjort, H.-E. Angstrom, Divided exhaust period: Effects of changing the relation between intake, blowdown and scavenging valve area, SAE Int. J. Engines 6 (2) (2013) 2013

[6] M. Masi, A. Toffolo, M. Antonello, Experimental analysis of a motorbike high speed racing engine, Applied Energy 87 (5) (2010) 1641 - 1650.

[7] G. Fontana, E. Galloni, Variable valve timing for fuel economy improvement in a small spark-ignition engine, Applied Energy 86 (1) (2009) 96-105.

[8] L. Cornolti, A. Onorati, T. Cerri, G. Montenegro, F. Piscaglia, 1d simulation of a turbocharged diesel engine with comparison of short and long egr route solutions, Applied Energy 111 (2013) $1-15$.

[9] M. Padzillah, S. Rajoo, R. Martinez-Botas, Influence of speed and frequency towards the automotive turbocharger turbine performance under pulsating flow conditions, Energy Conversion and Management 80 (0) (2014) 416 - 428.

[10] M. Chiong, S. Rajoo, A. Romagnoli, A. Costall, R. MartinezBotas, Integration of meanline and one-dimensional methods for prediction of pulsating performance of a turbocharger turbine, Energy Conversion and Management 81 (0) (2014) 270 - 281.

[11] F. S. Ahmed, S. Laghrouche, A. Mehmood, M. E. Bagdouri, Estimation of exhaust gas aerodynamic force on the variable geometry turbocharger actuator: 1d flow model approach, Energy Conversion and Management 84 (0) (2014) 436 - 447.

[12] R. Fleck, A. Cartwright, Coefficients of discharge in high performance two-stroke engines., SAE transactions 105 (3) (1996) 2469-2480.

[13] J. Decker, Critical and steady flow analysis of a high performance automotive exhaust port, $\mathrm{Ph} . \mathrm{D}$. thesis, University of Miami, Coral Gables, Florida, USA (2013).

[14] G. Trengrouse, B. Imrie, D. Mal, Comparison of unsteady flow discharge coefficients for sharp-edged orifices with steady flow values, Journal of Mechanical Engineering Science 8 (3) (1966) 322-329.

[15] S. Bohac, K. Landfahrer, Effects of pulsating flow on exhaust port flow coefficients, SAE Int. J. Engines (1999-01-0214).

[16] D. E. Winterbone, D. R. J. Pearson, Design techniques for engine manifolds: wave action methods for IC engines, Society of Automotive Engineers, 1999.

[17] R. Melli, E. Sciubba, C. Toro, An application of the proper orthogonal decomposition method to the thermo-economic optimization of a dual pressure, combined cycle powerplant, Energy Conversion and Management (0) (2014) -.

[18] H. Mezher, D. Chalet, J. Migaud, P. Chesse, Frequency based approach for simulating pressure waves at the inlet of internal combustion engines using a parameterized model, Applied Energy 106 (2013) 275-286.

[19] T. R. Smith, J. Moehlis, P. Holmes, Low-dimensional modelling of turbulence using the proper orthogonal decomposition: a tutorial, Nonlinear Dynamics 41 (1-3) (2005) 275-307.

[20] A. Chatterjee, An introduction to the proper orthogonal decomposition, Current Science 78 (7) (2000) 808-817.

[21] P. Moin, R. Moser, Characteristic-eddy decomposition of turbu- lence in a channel, Journal of Fluid Mechanics 200 (41) (1989) 509.

[22] W. Cazemier, R. Verstappen, A. Veldman, Proper orthogonal decomposition and low-dimensional models for driven cavity flows, Physics of fluids 10 (1998) 1685.

[23] P. J. Schmid, Dynamic mode decomposition of numerical and experimental data, J. Fluid Mech. 656 (2010) 5-28.

[24] J. W. Harrington, Visualization of the flow of exhaust through an internal combustion engine exhaust port, $\mathrm{Ph} . \mathrm{D}$. thesis, Massachusetts Institute of Technology, Department of Mechanical Engineering (1979).

[25] H. Dembinski, H.-E. Ångström, Swirl and injection pressure effect on post-oxidation flow pattern evaluated with combustion image velocimetry, CIV, and CFD simulation, SAE Technical Paper 2013-01-2577.

[26] K. Tanaka, Air flow through exhaust valve of conical seat., in: Proceedings of Third International Congress for Applied Mechanics, Vol. 1, 1931, pp. 287-295.

[27] G. L. Brown, A. Roshko, et al., On density effects and large structure in turbulent mixing layers, Journal of Fluid Mechanics 64 (4) (1974) 775-816.

[28] S. Gaikwad, K. Arora, V. Korivi, S. K. Cho, Steady and transient CFD approach for port optimization, SAE International Journal of Materials and Manufacturing 1 (1) (2008) 754-762.

[29] B. Leonard, Simple high-accuracy resolution program for convective modelling of discontinuities, International Journal for Numerical Methods in Fluids 8 (10) (1988) 1291-1318.

[30] Y. Wang, B. Semlitsch, M. Mihaescu, L. Fuchs, Flow structures and losses in the exhaust port of an internal combustion engine, in: Proceedings of the ASME 2013 International Mechanical Engineering Congress \& Exposition, no. IMECE 2013-64610, ASME, 2013.

[31] B. Semlitsch, V. JyothishKumar, M. Mihaescu, L. Fuchs, E. Gutmark, M. Gancedo, Numerical flow analysis of a centrifugal compressor with ported and without ported shroud, SAE Technical Paper 2014-01-1655.

[32] P. Holmes, J. L. Lumley, G. Berkooz, Turbulence, coherent structures, dynamical systems and symmetry, Cambridge university press, 1998.

[33] S. Berger, L. Talbot, L. Yao, Flow in curved pipes, Annual Review of Fluid Mechanics 15 (1) (1983) 461-512.

[34] D. Palfreyman, R. Martinez-Botas, The pulsating flow field in a mixed flow turbocharger turbine: an experimental and computational study, Journal of turbomachinery 127 (1) (2005) 144-155. 